\title{
Red Blood Cells Motion in a Glass Microchannel
}

\author{
Diana Pinho*, Ana I. Pereira ${ }^{* \dagger}$ and Rui Lima*,** \\ * Polytechnic Institute of Bragança, Portugal \\ ${ }^{\dagger}$ Algoritmi, University of Minho, Portugal \\ ** CEFT, FEUP, Porto University, Portugal
}

\begin{abstract}
The motion of the red blood cells (RBCs) flowing in microvessels and microchannels depend on several effects, such as hematocrit (Hct), geometry, and temperature. According to our knowledge, the effect of the temperature on RBC motion was never investigated at a microscale level. Hence, the aim of the present work is to determine the effect of the temperature on the RBC's trajectories and to investigate the best approximation of the trajectories through a nonlinear optimization. In vitro human blood was pumped through a $100 \mu \mathrm{m}$ circular microchannel and by using a confocal microPTV system the RBC's trajectories were measured at different temperatures, i.e., $25^{\circ} \mathrm{C}$ and $37^{\circ} \mathrm{C}$. In this study we measured the motion of forty cells flowing in the middle of the microchannel and applied different functions to approximate its behavior.
\end{abstract}

Keywords: Red Blood Cells. Nonlinear Optimization. Biomicrofluidics. Microcirculation.

PACS: $02.60 . \mathrm{Pn}$

\section{INTRODUCTION}

Blood is a fluid composed of a suspension of cells, proteins and ions in plasma. In normal blood, three types of cells comprise about $46 \%$ of its volume. These cells are the red blood cells (also known as erythrocytes), white blood cells (also known as leukocytes) and platelets (also known as thrombocytes). Figure 1 shows different kinds of human blood cells.

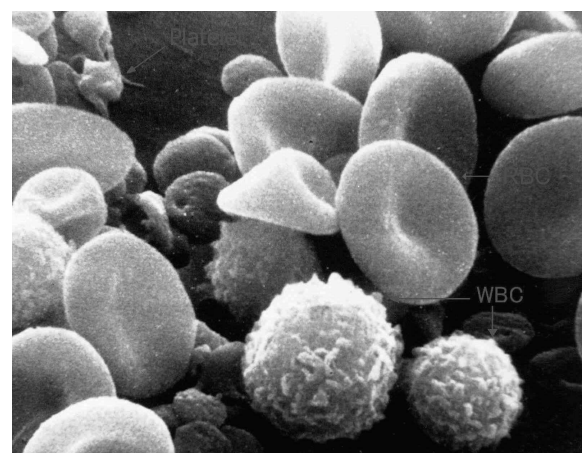

FIGURE 1. Scanning electron microscope image of human blood cells (adapted from [13]).

In the microcirculation, the flow behavior of RBCs plays a crucial role in many physiological and pathological phenomena. For example, the random-like transverse motion and rotation of RBCs in shear flow is believed to play an important role in thrombogenesis $[3,10]$. As a consequence, many rheological studies have been performed on both microvessels and microchannels to investigate the effect of the hematocrit (Hct), geometry, and temperature on the RBCs flowing behavior [2, 3, 10]. Recently Lima and his colleagues measured the red blood cells (RBCs) radial dispersion (Dyy) in both glass [6, 7] and polydimensiloxane (PDMS) [8] microchannels by using a confocal microPTV system. By comparing Lima et al. results with the measurements performed by Goldsmith and his colleagues [3] several quantitative deviations were observed between both experimental results. One possible reason for observed discrepancies may due to the different temperatures used in the two cases, i.e., Lima et al. used body temperatures $\left(37^{\circ} \mathrm{C}\right)$ whereas Goldsmith et al. used room temperatures. Hence, it is important to investigate the effect of the temperature on the RBC motion. The experiments were performed in the middle of $100 \mu \mathrm{m}$ glass capillaries at temperatures of $25^{\circ} \mathrm{C}$ and $37^{\circ} \mathrm{C}$, by using a confocal micro-PTV system. In the present study, the trajectories of forty 
RBCs were measured and different functions were applied with the purpose to find the one that best approximate to its flow behavior.

\section{MATERIALS AND METHODS}

In the present study we used Dextran 40 (Dx-40; Otsuka Medicine, Tokyo, Japan) containing $12 \pm 2 \%$ (12Hct) of human RBCs. The RBCs were labeled with a lipophilic carbocyanime derivative, chloromethylbenzamido (CM-Dil, C7000, Molecular Probes, Eugene, OR, USA). A detailed description about the procedure for labeling the human RBCs can be found elsewhere [7]. Additionally, we used a $100 \mu \mathrm{m}$ circular borosilicate glass microchannel fabricated by Vitrocom (Mountain Lakes, NJ, USA). The microchannel was mounted on a slide glass with a thickness of $80 \pm 20 \mu \mathrm{m}$ and was immersed in glycerol to minimize the refraction from the walls.

The confocal micro-PTV system used in this study consists of an inverted microscope (IX71; Olympus, Japan) combined with a confocal scanning unit (CSU22; Yokogawa, Japan), a diode-pumped solid-state (DPSS) laser (Laser Quantum, UK) with an excitation wavelength of 532nm and a high-speed camera (Phantom v7.1; Vision Research, USA) (see Figure2). The microchannels were placed on the stage of the inverted microscope and by using a syringe pump (KD Scientific, USA) a pressure-driven flow was kept constant $(\operatorname{Re} \approx 0.008)$. Additionally, by using a thermo plate controller (Tokai Hit) it was possible to apply different temperatures to the surrounding environment, i.e., $25^{\circ} \mathrm{C} \pm 1$ and $37^{\circ} \mathrm{C} \pm 1$. More detailed information about this system can be found elsewhere [4, 5, 7].

FIGURE 2. Confocal micro-PTV experimental set-up and trajectory of labeled RBC through a circular glass microchannel.

The confocal images were captured in middle of the capillary through a dry $40 \times$ objective lens at a rate of 100 frames/s. A manual tracking plugin (MTrackJ) [11] of an image analysis software (Image J, NIH) [1] was used to track individual RBC. By using MTrackJ plugin, the bright centroid of the selected RBC was automatically computed through successive images for an interval of time of $10 \mathrm{~ms}$. After obtaining series of $\mathrm{x}$ and y positions, data were exported for the determination of each individual RBC trajectory and to analyse the best mathematical function that approximates to the RBCs experimental flow behavior.

In this study it was observed and analyzed forty cells: twenty at temperature $25^{\circ} \mathrm{C}$ and other twenty cells at the temperature $37^{\circ} \mathrm{C}$. For each cell $i$, and using MTrackJ plugin system, we obtain $\left\{\left(x_{j}, y_{j}\right), j=1, \ldots, k_{i}\right\}$ data. In this experiences $k_{i}$ can assume values between 23 and 195. The aim of this work is to obtain a better approximation for the data using nonlinear optimization [12]. For that we consider three different functions (polynomials, sum of trigonometric functions and a sum of exponential functions) defined as:

$$
f_{1}(x, p)=\sum_{i=0}^{9} p_{i} x^{i} ; \quad f_{2}(x, a, b)=a_{0}+\sum_{i=1}^{8} a_{i} \cos (i x)+b_{i} \sin (i x) ; \text { and } \quad f_{3}(x, c, d, g)=\sum_{i=1}^{8} g_{i} e^{\left(-\left(\frac{\left(x-c_{i}\right)}{d_{i}}\right)^{2}\right)}
$$

where $p \in \mathbb{R}^{10}, a \in \mathbb{R}^{9}, b, c, d, g \in \mathbb{R}^{8}$ are the function parameters and the vector $x \in \mathbb{R}^{k_{i}}$, where $i$ represents the cell number. To identify the functions parameters it was used the tool cftool present in Curve Fitting Toolbox from Matlab [9].

\section{RESULTS AND DISCUSSION}

The error of nonlinear least squares approximation of the selected cells are listed in the Table 1, where Cell refers to the cell number, $F_{i}=\sum_{j=1}^{k_{i}}\left(y_{j}-f_{i}\left(x_{j}\right)\right)^{2}$ is the nonlinear least squares approximation error of the function $f_{i}(x)$, with $i=1,2,3$, Av refers the error average, and $\mathbf{s}$ corresponds to the standard deviation of the errors.

The results, from Table 1, indicate that the function $f_{2}(x, a, b)$ (sum of trigonometric functions) was the best approximation to the motion of the cells in the microchannel. Only in five cells (three in the with temperature $25^{\circ} \mathrm{C}$ and two with the temperature $37^{\circ} \mathrm{C}$ ) we have obtained a better performance with the function $f_{3}(x, a, b, c)$. This is confirmed by the value of the error average. Another important aspect is the fact that the standard deviation of the 
TABLE 1. Numerical Results obtained using cftool.

\begin{tabular}{cccccccc}
\hline \multicolumn{3}{c}{ Cells with temperature $25^{\circ} C$} & \multicolumn{3}{c}{ Cells with temperature $37^{\circ} C$} \\
\hline Cell & $F_{1}$ & $F_{2}$ & $F_{3}$ & Cell & $F_{1}$ & $F_{2}$ & $F_{3}$ \\
\hline $\mathbf{1}$ & 3.453 & 2.537 & 2.701 & $\mathbf{2 1}$ & 0.792 & 0.199 & 0.485 \\
$\mathbf{2}$ & 1.707 & 0.834 & 2.363 & $\mathbf{2 2}$ & 0.994 & 0.497 & 0.985 \\
$\mathbf{3}$ & 3.258 & 2.282 & 1.644 & $\mathbf{2 3}$ & 2.898 & 1.664 & 1.230 \\
$\mathbf{4}$ & 1.484 & 0.720 & 1.066 & $\mathbf{2 4}$ & 0.222 & 0.099 & 0.217 \\
$\mathbf{5}$ & 1.146 & 0.117 & 1.121 & $\mathbf{2 5}$ & 0.660 & 0.328 & 0.409 \\
$\mathbf{6}$ & 3.937 & 0.938 & 3.677 & $\mathbf{2 6}$ & 0.876 & 0.331 & 0.513 \\
$\mathbf{7}$ & 1.756 & 1.256 & 1.565 & $\mathbf{2 7}$ & 2.072 & 0.569 & 1.243 \\
$\mathbf{8}$ & 2.521 & 1.005 & 1.343 & $\mathbf{2 8}$ & 0.175 & 0.116 & 0.157 \\
$\mathbf{9}$ & 0.237 & 0.056 & 0.189 & $\mathbf{2 9}$ & 1.999 & 1.030 & 1.769 \\
$\mathbf{1 0}$ & 1.757 & 0.820 & 1.028 & $\mathbf{3 0}$ & 1.572 & 0.849 & 0.629 \\
$\mathbf{1 1}$ & 1.868 & 0.865 & 0.941 & $\mathbf{3 1}$ & 7.461 & 3.477 & 4.180 \\
$\mathbf{1 2}$ & 1.400 & 0.482 & 0.631 & $\mathbf{3 2}$ & 1.703 & 0.789 & 1.070 \\
$\mathbf{1 3}$ & 1.574 & 0.899 & 1.342 & $\mathbf{3 3}$ & 7.486 & 2.832 & 7.572 \\
$\mathbf{1 4}$ & 3.684 & 2.421 & 2.772 & $\mathbf{3 4}$ & 2.855 & 0.569 & 2.123 \\
$\mathbf{1 5}$ & 0.514 & 0.222 & 0.255 & $\mathbf{3 5}$ & 1.089 & 0.659 & 1.667 \\
$\mathbf{1 6}$ & 1.321 & 0.597 & 0.808 & $\mathbf{3 6}$ & 5.204 & 1.949 & 2.004 \\
$\mathbf{1 7}$ & 4.010 & 3.342 & 2.169 & $\mathbf{3 7}$ & 3.223 & 0.712 & 9.552 \\
$\mathbf{1 8}$ & 2.426 & 1.782 & 2.017 & $\mathbf{3 8}$ & 1.729 & 0.585 & 0.761 \\
$\mathbf{1 9}$ & 0.650 & 0.354 & 1.061 & $\mathbf{3 9}$ & 1.318 & 0.605 & 0.828 \\
$\mathbf{2 0}$ & 4.353 & 2.391 & 1.95 & $\mathbf{4 0}$ & 1.963 & 0.862 & 1.864 \\
\hline Av & 2.153 & 1.196 & 1.532 & Av & 2.315 & 0.936 & 1.963 \\
$\mathbf{s}$ & 1.240 & 0.936 & 0.891 & $\mathbf{s}$ & 2.114 & 0.892 & 2.454 \\
\hline
\end{tabular}

errors is small when we use the function $f_{2}$. Two examples of the calculated functions, $f_{1}, f_{2}$ and $f_{3}$ can be observed in the Figure 3.
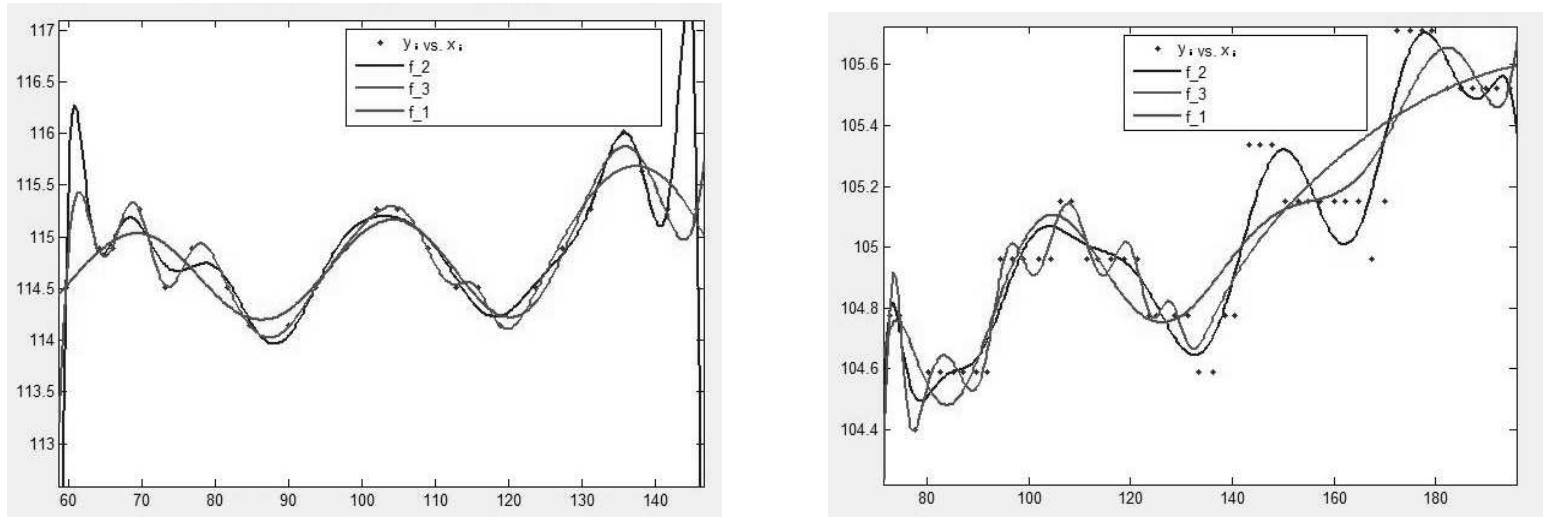

FIGURE 3. Cell number 15 at $\mathrm{T}=25^{\circ} \mathrm{C}$ and cell number 2 at $\mathrm{T}=37^{\circ} \mathrm{C}$.

The preliminary results, suggest that the movement of RBCs along the microchannel follow a behavior equivalent to the sum of trigonometric functions.

\section{CONCLUSIONS}

In this work we measure the motion of RBCs along the middle of a microchannel. The results were approximated by three different types of functions applying nonlinear least squares theory.

The results obtained from this preliminary study, indicates that the function that has a better approximation to the data is based on trigonometric functions. In future work we will consider different type of functions and a bigger amount of cells with different temperatures and Hcts. 
An on going study to obtain more detailed quantitative measurements of the blood flow behavior through a glass microchannel is currently under way.

\section{ACKNOWLEDGMENT}

This study was supported in part by the following grants: Grant-in-Aid for Science and Technology (PTDC/SAUBEB/108728/2008, PTDC/SAU-BEB/105650/2008 and PTDC/EME-MFE/099109/2008) from the Science and Technology Foundation (FCT) and COMPETE, Portugal.

\section{REFERENCES}

1. M. Abramoff , P. Magelhaes and S. Ram, Image processing with image J, Biophotonics Int. 11, 2004, pp. $36-42$.

2. S. Chien, S. Usami and R. Skalak, Blood flow in small tubes, Handbook of Physiology - The Cardiovascular System IV, 1984 , pp. $217-249$.

3. H. Goldsmith and V. Turitto, Rheological aspects of thrombosis and haemostasis: basic principles and applications, ICTH Report - Subcommittee on Rheology of the International committee on thrombosis and haemostasis, 55, 1986, pp. 415 - 435.

4. R. Lima, S. Wada, K. Tsubota, M. Takeda, and T. Yamaguchi, Confocal micro-PIV measurements of three dimensional profiles of cell suspension flow in a square microchannel, Measurement Science and Technology 17, 2006, pp. 797-808.

5. R. Lima, S. Wada, S. Tanaka, M. Takeda, T. Ishikawa, K. Tsubota, Y. Imai and T. Yamaguchi, In vitro blood flow in a rectangular PDMS microchannel: experimental observations using a confocal micro-PIV system, Biomedical Microdevices 10, 2008, pp. 153-67.

6. R. Lima, T. Ishikawa, Y. Imai, M. Takeda, S. Wada, and T. Yamaguchi, Radial dispersion of red blood cells in blood flowing through glass capillaries: role of hematocrit and geometry, Journal of Biomechanics 41, 2008, pp. 2188 - 2196.

7. R. Lima, T. Ishikawa, Y. Imai, M. Takeda, S. Wada, and T. Yamaguchi, Measurement of individual red blood cell motions under high hematocrit conditions using a confocal micro-PTV system, Annals of Biomedical Engineering 37, 2009, pp. 1546 -1559 .

8. R. Lima, M.S.N. Oliveira, T. Ishikawa, H. Kaji, S. Tanaka, M. Nishizawa and T. Yamaguchi, Axisymmetric PDMS microchannels for in vitro haemodynamics studies, Biofabrication 1, 2009, 035005.

9. MathWorks, Optimization Toolbox User's Guide, 2010.

10. G. Mchedlishvili and N. Maeda, Blood flow structure related to red cell flow: a determination of blood fluidity in narrow microvessels, Japanese Journal of Physiology 51, 2001, pp. 19-30.

11. E. Meijering, I. Smal and G. Danuser, Tracking in molecular bioimaging, IEEE Signal Process. Mag. 23, 2006, 46-53.

12. J. Nocedal, S. Wright, Numerical Optimization. Springer Series in Operations Research, Springer, New York, 1999.

13. B. Wetzel. and H. Schaefer, National Cancer Institute, Human Blood Cells, 1982. Available from: http://en.wikipedia.org/wiki/Wikipedia:Featured_picture_candidates/Human_Blood_Cells. 